Korean J. Math. 19 (2011), No. 4, pp. 409-421

\title{
THE HOMOLOGY HOMOMORPHISM INDUCED BY HARER MAP
}

\author{
Deoguu Lee And Yonguin SONG*
}

\begin{abstract}
We study a natural map from the braid group to the mapping class group which is called Harer map. It is rather new and different from the classical map which was studied in 1980's by F. Cohen, J. Harer et al. We show that this map is homologically trivial for most coefficients by using the fact that this map factors through the symmetric group.
\end{abstract}

\section{Introduction}

The classical Harer map is an obvious map from braid groups to mapping class groups. In the latter group there are plenty of braid relations among Dehn twists. In this paper we introduce a new map from the braid group to the mapping class group which is also naturally defined. We call this map a Harer map throughout this paper.

The construction of a Harer map is made by identifying the braid group as a subgroup of the mapping class group of a genus zero surface with boundary components as follows. Let $S_{0, k+1}$ be a sphere with $k+1$ disks removed and parametrized boundary circles $\partial_{0}, \partial_{1}, \ldots, \partial_{k}$. Consider the orientation preserving diffeomorphisms that fix the first boundary component $\partial_{0}$ pointwise but may permute the other $k$ boundary components as long as they preserve the parametrization of each. The associated mapping class group $\Gamma_{0,(k), 1}$ is the ribbon braid group

Received October 20, 2011. Revised November 21, 2011. Accepted November 30, 2011.

2000 Mathematics Subject Classification: 20F36, 20F28, 55R37.

Key words and phrases: braid groups, automorphism groups of groups, mapping class groups.

The second author was supported by National Research Foundation of Korea (NRF-2010-0011314).

${ }^{*}$ Corresponding author. 
$R B_{k}$ on $k$ ribbons. $R B_{k}$ is the wreath product $B_{k}<\mathbb{Z}$, and $B_{k}$ can naturally be identified as a subgroup.

$$
\Gamma_{0,1}^{k} \simeq B_{k} \subset B_{k} \prec \mathbb{Z}=R B_{k} \simeq \Gamma_{0,(k), 1} .
$$

Thus this identification leads to homomorphisms of the braid group into the mapping class group. Consider two copies of the surface $S_{0, k+1}$ glued along their boundary components $\partial_{1}, \ldots, \partial_{k}$ to form a surface $S_{k-1,2}$.

Any diffeomorphism of $S_{0, k+1}$ as described above can be extended to $S_{k-1,2}$ by "mirroring" the action on the second copy of $S_{0, k+1}$ and can then be extended to $S_{g+k, 2}$ by the identity diffeomorphism.

This gives rise to a Harer map:

$$
\text { Harer : } B_{k} \stackrel{m}{\longrightarrow} B_{k} \times_{\Sigma_{k}} B_{k} \stackrel{\alpha}{\longrightarrow} \Gamma_{g+k, 2},
$$

where $m$ is mirroring, $\alpha$ is induced by gluing $S_{g+1,2}$ on the right boundary component of $S_{k-1,2}$, and the group in the middle is defined as the pullback in the following diagram:

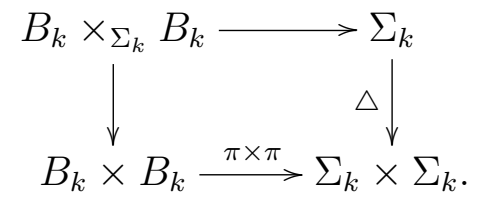

The main result of this paper is to prove that the Harer map induces zero homomorphism on homology in some coefficients.

MAIN TheOREM. The homology homomorphism Harer ${ }_{*}: H_{*}\left(B_{k} ; \mathbb{F}\right)$ $\rightarrow H_{*}\left(\Gamma_{g+k, 2} ; \mathbb{F}\right)$ is zero for $0<*<\frac{g+k}{2}$ and $\mathbb{F}=\mathbb{Q}, \mathbb{Z}_{p}(p \neq 2$ is prime $)$. Hence the map Harer $* H_{*}\left(B_{\infty} ; \mathbb{F}\right) \rightarrow H_{*}\left(\Gamma_{\infty, 1} ; \mathbb{F}\right)$ is zero.

In the proof of Main theorem we use the fact that a Harer map factors through the symmetric group and the homology of symmetric group is isomorphic to $\mathbb{Z}_{2}$. The second part of Main Theorem follows by the homology stabilization theorem for mapping class groups.

\section{Mapping class groups and homology stabilization theorem}

Let $S_{g, n+1}^{k}$ denote an oriented smooth surface of genus $g$ with $k$ marked points specified and $n+1$ boundary components. The mapping class group $\Gamma_{g, n+1}^{k}$ is defined to be the group of isotopy classes of orientation preserving self-diffeomorphisms of $S_{g, n+1}^{k}$ which fix the $k$ points 
pointwise, and are identity on the boundary. If $k$ is zero, we denote $S_{g, n+1}:=S_{g, n+1}^{0}$ and $\Gamma_{g, n+1}:=\Gamma_{g, n+1}^{0}$. By gluing a torus with two boundary components to one of the boundary components of $S_{g, n+1}^{k}$, we get a surface $S_{g+1, n+1}^{k}$ (See Figure 1).

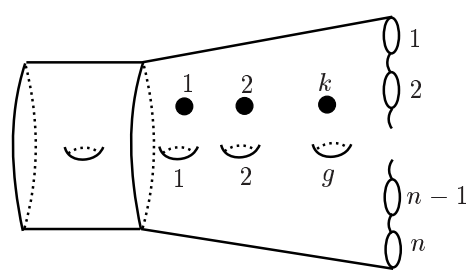

FIGURE 1. $S_{g, n+1}^{k} \longrightarrow S_{g+1, n+1}^{k}$

Extending diffeomorphisms by the identity induces a map of mapping class groups

$$
\Gamma_{g, n+1}^{k} \rightarrow \Gamma_{g+1, n+1}^{k}
$$

and we may define the associated stable mapping class group

$$
\Gamma_{\infty, n}^{k}=\lim _{g \rightarrow \infty} \Gamma_{g, n+1}^{k}
$$

The $n$ boundary components not used in this process will be called free. Consider diffeomorphisms that may permute free boundary components. More precisely, the boundary components should be thought of as having a parametrization and diffeomorphisms have to be compatible with these. The associated mapping class groups will be denoted by $\Gamma_{g,(n), 1}^{k}$. There are normal extensions and stable maps:

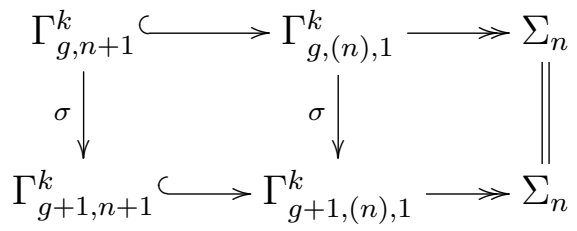

with quotients of the symmetric group $\Sigma_{n}$. Thus we have the map of associated stable mapping class groups

$$
\Gamma_{\infty, n}^{k}=\lim _{g \rightarrow \infty} \Gamma_{g, n+1}^{k} \hookrightarrow \Gamma_{\infty,(n)}^{k}=\lim _{g \rightarrow \infty} \Gamma_{g,(n), 1}^{k} \rightarrow \Sigma_{n} .
$$

Let $A: S_{g, r} \rightarrow S_{g, r+1}(r \geq 1)$ and $B: S_{g, r} \rightarrow S_{g+1, r-1}(r \geq 2)$ be the inclusions defined by adding a pair of pants (a copy of $S_{0,3}$ ) sewn 
along one boundary components for $A$ and two boundary components for $B$. Also define $C: S_{g, r} \rightarrow S_{g+1, r-2}(r \geq 2)$ by gluing two boundary components together.

Theorem 2.1 (Harer Stability Theorem, [5]). The associated homomorphisms of mapping class groups defined by the maps $A, B, C$ induce isomorphisms of integral homology:

$$
A_{*}: H_{n}\left(\Gamma_{g, r}\right) \rightarrow H_{n}\left(\Gamma_{g, r+1}\right)
$$

for $n>1$ when $g \geq 3 n-2, r \geq 1$, and for $n=1$, when $g \geq 2, r \geq 1$,

$$
B_{*}: H_{n}\left(\Gamma_{g, r}\right) \rightarrow H_{n}\left(\Gamma_{g+1, r-1}\right)
$$

for $n>1$ when $g \geq 3 n-1, r \geq 2$, and for $n=1$, when $g \geq 3, r \geq 2$,

$$
C_{*}: H_{n}\left(\Gamma_{g, r}\right) \rightarrow H_{n}\left(\Gamma_{g+1, r-2}\right)
$$

when $g \geq 3 n, r \geq 2$.

Corollary 2.2. $H_{*}\left(\Gamma_{g, r}\right)$ is independent of $g$ and $r$ in degree $*<\frac{g-1}{3}$.

In [8], Ivanov improves this to the case when the genus of $S_{g, r}$ is at least $2 n+1$.

There are group maps

$$
\Gamma_{g, r} \rightarrow \Gamma_{g, r-1} \text { and } \Gamma_{g, r} \rightarrow \Gamma_{g+1, r}
$$

induced by gluing a disk, a torus with two boundary components to one of the boundary components of $S_{g, r}$, respectively. By the theorem of Harer and Ivanov $[5,8]$, these maps induce isomorphisms in $H_{*}(\cdot ; \mathbb{Z})$ for $* \leq \frac{g-1}{2}$, and thus there is a stable range in which the group homology $H_{*}\left(\Gamma_{g, r} ; \mathbb{Z}\right)$ is independent of $g$ and $r$. In this range it agrees with $H_{*}\left(\Gamma_{\infty} ; \mathbb{Z}\right)$ where $\Gamma_{\infty}=\lim _{g \rightarrow \infty} \Gamma_{g, 1}$ is the stable mapping class group:

$$
H_{*}\left(\Gamma_{g, 1} ; \mathbb{Z}\right) \stackrel{\simeq}{\longrightarrow} H_{*}\left(\Gamma_{\infty} ; \mathbb{Z}\right) \quad \text { for } g \geq 2 *+1 \text {. }
$$

Let $F_{n}=\left\langle a_{1}, \ldots, a_{n}\right\rangle$ be the free group on $n$ generators and let $\operatorname{Aut} F_{n}$ be its automorphism group. Let $\Sigma_{n}$ be the symmetric group and let $\varphi_{n}$ : $\Sigma_{n} \rightarrow \operatorname{Aut} F_{n}$ be the homomorphism that to a permutation $\sigma$ associates the automorphism $\varphi_{n}(\sigma): a_{i} \mapsto a_{\sigma(i)}$.

Recently, Galatius proved the following theorem in [4]:

THEOREM 2.3. $\varphi_{n}$ induces an isomorphism

$$
\left(\varphi_{n}\right)_{*}: H_{*}\left(\Sigma_{n}\right) \longrightarrow H_{*}\left(\operatorname{Aut} F_{n}\right)
$$

for $n \geq 2 *+2$. 
The homology groups are independent of $n$ in the sense that increasing $n$ induces isomorphisms $H_{*}\left(\Sigma_{n}\right) \simeq H_{*}\left(\Sigma_{n+1}\right)$ and $H_{*}\left(\operatorname{Aut} F_{n}\right) \simeq$ $H_{*}\left(\operatorname{Aut} F_{n+1}\right)$ when $n \geq 2 *+2([6,7,9])$. Since $\Sigma_{n}$ is a finite group, with rational coefficients the homology groups vanish. Thus we have the following corollary.

COROLlary 2.4. The groups

$$
H_{*}\left(\Sigma_{n} ; \mathbb{Q}\right)=H_{*}\left(\operatorname{Aut} F_{n} ; \mathbb{Q}\right)=0
$$

for $n \geq 2 *+2$.

Let $B_{n}$ be the braid group on $n$ strings. Artin [1] identified $B_{n}$ as a subgroup of Aut $F_{n}$ as follows. Let $\sigma_{i} \in B_{n}$ denote a standard generator which crosses the $i$ th over the $(i+1)$ st string. Artin's map

$$
\phi: B_{n} \rightarrow \operatorname{Aut} F_{n}
$$

is defined by taking $\sigma_{i}$ to the automorphism

$$
\phi\left(\sigma_{i}\right): a_{j} \rightarrow \begin{cases}a_{j} & \text { if } j \neq i, i+1 \\ a_{i+1} & \text { if } j=i \\ a_{i+1}^{-1} a_{i} a_{i+1} & \text { if } j=i+1\end{cases}
$$

The map $\phi$ extends to a map:

$$
B_{\infty}:=\lim _{n \rightarrow \infty} B_{n} \rightarrow \operatorname{Aut} F_{\infty}=\lim _{n \rightarrow \infty} \operatorname{Aut} F_{n} .
$$

Tillmann [12] proved the following theorem:

Theorem $2.5([12$, Theorem 1$]) . \phi_{*}: H_{*}\left(B_{\infty} ; \mathbb{F}\right) \rightarrow H_{*}\left(\right.$ Aut $\left.F_{\infty} ; \mathbb{F}\right)$ is trivial when $\mathbb{F}=\mathbb{Q}$ or $\mathbb{F}=\mathbb{Z}_{p}$ for any odd prime $p$.

An element of the symmetric group $\Sigma_{n}$ acts naturally by permutations of the generators on $F_{n}$. The composition with the natural surjection $\pi$ from the braid group to the symmetric group defines the homomorphism

$$
B_{n} \stackrel{\pi}{\longrightarrow} \Sigma_{n} \stackrel{\varphi_{n}}{\longrightarrow} \operatorname{Aut} F_{n},
$$

where $\pi\left(\sigma_{i}\right)=(i, i+1)$ for generators $\sigma_{i}$ of $B_{n}$. As $\phi, \varphi_{n} \circ \pi$ also commutes with limits and extends to a map of stable groups. Although these maps are very different, they induce the same map on homology [12].

Theorem $2.6([12$, Theorem 2$]) . \phi_{*}=(\varphi \circ \pi)_{*}: H_{*}\left(B_{\infty} ; \mathbb{F}\right) \rightarrow$ $H_{*}\left(\right.$ Aut $\left.F_{\infty} ; \mathbb{F}\right)$ when $\mathbb{F}=\mathbb{Q}$ or $\mathbb{F}=\mathbb{Z}_{p}$ for any odd prime $p$. 
2.1. Splitting Theorem. In this section, we describe a splitting theorem [13] which plays a key role in the proof of Main Theorem.

A group $G$ is perfect if every element can be written as a product of commutators, that is, $[G, G]=G$. Any group $G$ has a unique maximal perfect subgroup which we will denote by $P(G)$. Since the homomorphic image of a perfect group is also perfect, $P(G)$ is a characteristic subgroup of $G$.

Recall that a group $G$ is called a direct sum group if there is a homomorphism $\oplus: G \times G \rightarrow G$.

We consider the more general case than direct sum group.

Definition 2.7. Two groups $G$ and $H$ form a direct sum pair if $H<G$ and there is a homomorphism $\oplus: H \times G \rightarrow G$ such that for any $g_{1}, \ldots, g_{s} \in G$ and $h_{1}, \ldots, h_{s} \in H$ there exist elements $c \in P(G)$ and $d \in P(H)$ satisfying the following:

$$
1 \oplus g_{1}=c g_{i} c^{-1} \text { and } h_{i} \oplus 1=d h_{i} d^{-1} \text { for all } i=1, \ldots, s .
$$

Theorem 2.8 ([2, Proposition 5.2]). $B G^{+}$admits a left $H$-action by $B \mathrm{H}^{+}$where $B(\cdot)^{+}$means the Qullen's plus-construction of the classifying space of groups.

Proof. Note that $(B H \times B G)^{+}=B H^{+} \times B G^{+}$. Thus the direct sum homomorphism $\oplus$ induces a map

$$
B \oplus^{+}: B H^{+} \times B G^{+} \rightarrow B G^{+} .
$$

Let $*$ denote the basepoint of $B G^{+}$and $B H^{+}$. The map $B \oplus^{+}\left({ }_{-}, *\right)$ : $B H^{+} \rightarrow B G^{+}$is induced by $\oplus 1$. By $(2.2),{ }_{-} \oplus 1$ factors through $H$. We show that the induced map $f: B H^{+} \rightarrow B H^{+}$is a homotopy equivalence. Since $P(H) \triangleleft H, B P(H)$ is a regular cover of $B H$, and hence $B P(H)^{+}$ is the universal cover of $B H^{+}$. By $(2.2)$, the map $B P(H)^{+} \rightarrow B P(H)^{+}$ induced by $f$ is the identity on homology [13, Lemma 1.3]. Hence, by the Whitehead theorem, it is a homotopy equivalence. Also, $f$ is a homotopy equivalence. Similarly, $B \oplus^{+}\left(*_{-}\right)$is a homotopy equivalence of $B G^{+}$. Choose homotopy inverses $r$ and $t$ for these two maps. Then $\mu=B \oplus^{+} \circ(r \times t): B H^{+} \times B G^{+} \rightarrow B G^{+}$defines an H-action.

REMARK 2.9. Theorem 2.8 means there is a map $\mu: B H^{+} \times B G^{+} \rightarrow$ $B G^{+}$such that $\left.\mu\right|_{B H^{+}}$is homotopic to the map induced by the inclusion $H \hookrightarrow G$ and $\left.\mu\right|_{B G^{+}}$is homotopic to the identity. When $H$ is equal to $G$, then $B G^{+}$is an H-space. 
Corollary 2.10 ([2, Corollary 5.3]). Assume $G$ and $H$ form a direct sum pair, and that there is a splitting homomorphism $l: G \rightarrow H$. Then $B G^{+} \simeq B H^{+} \times F$, where $F$ is the homotopy fiber of the map $B G^{+} \rightarrow B H^{+}$.

Proof. Let $F$ be the homotopy fiber of the map $B l^{+}: B G^{+} \rightarrow B H^{+}$, and let $j: F \rightarrow B G^{+}$denote the inclusion of the fiber. Define $B H^{+} \times$ $F \rightarrow B G^{+}$by mapping $(x, y)$ to $\mu(x, j(y))$. Because $\mu$ defines an $\mathrm{H}$ action, this induces an isomorphism on homotopy groups and hence is a homotopy equivalence.

Recall that for all $k$ and $g>2, \Gamma_{g, k}$ is perfect [10].

Consider the following exact sequence

$$
\Gamma_{g, k+1} \hookrightarrow \Gamma_{g,(k), 1} \stackrel{\rho}{\longrightarrow} \Sigma_{k} .
$$

Clearly, the kernel of $\rho$ is $\Gamma_{g, k}$. Since an extension of perfect groups is again perfect, we see $\rho^{-1}\left(P\left(\Sigma_{k}\right)\right)$ is perfect. In fact, it must be maximal. So we have

$$
P\left(\Gamma_{g,(k), 1}\right)=\rho^{-1}\left(P\left(\Sigma_{k}\right)\right)=\rho^{-1}\left(A_{k}\right) \text { for } g>2,
$$

where $A_{k}$ is an alternating subgroup of $\Sigma_{k}$. And in particular, $P\left(\Gamma_{g,(k), 1}\right)$ contains $\Gamma_{g, 1}$.

On the other hands, by extending diffeomorphisms by the identity on an attached disk $S_{0, k+1}$ with $k$ disks removed, we defines the inclusion map incl : $\Gamma_{g, 1} \rightarrow \Gamma_{g,(k), 1}$ (See Figure 2):

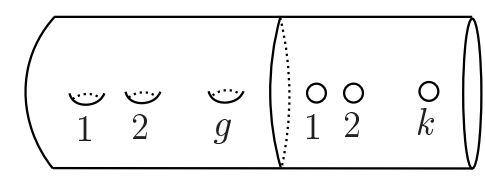

Figure 2. incl : $\Gamma_{g, 1} \longrightarrow \Gamma_{g,(k), 1}$

We may now also fill in the $k$ disks, and once again extend diffeomorphisms by the identity. This defines the forgetful map $l: \Gamma_{g,(k), 1} \rightarrow \Gamma_{g, 1}$ (See Figure 3):

Clearly, $l \circ$ incl is the identity homomorphism. As stabilization commutes with both incl and $l$, these homomorphisms extend to the stable mapping class groups. 


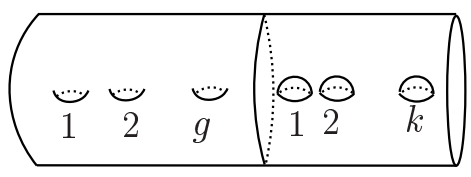

Figure $3 . l: \Gamma_{g,(k), 1} \longrightarrow \Gamma_{g, 1}$

The retraction induced by the group homomorphisms incl and $l$ on the Qullen's plus-construction of the classifying spaces is multiplicative, and hence gives rise to a splitting spaces. So we have the following splitting:

ThEOREM 2.11. $\left(B \Gamma_{\infty,(k)}\right)^{+} \simeq B \Gamma_{\infty}^{+} \times B \Sigma_{k}^{+}$.

Proof. Define $\oplus: \Gamma_{\infty} \times \Gamma_{\infty,(k)} \rightarrow \Gamma_{\infty,(k)}$ by letting $\Gamma_{\infty}$ act on the odd genus and $\Gamma_{\infty,(k)}$ act on the even genus. Then $\Gamma_{\infty,(k)}$ and $\Gamma_{\infty}$ form a direct sum pair. To check the property $(2.2)$, let $h_{1}, \ldots, h_{s} \in \Gamma_{\infty}$. Then they are in the image of $\Gamma_{g, 1}$ for some large $g$. Now choose an appropriate diffeomorphism of the surface $S_{2 g, 1} \subset S_{2 g, k+1}$ which moves the even genus over the odd genus to the last $g$ genus. Let $d \in \Gamma_{\infty}$ be its homotopy class. Similarly, let $g_{1}, \ldots, g_{s} \in \Gamma_{\infty,(k)}$. Then they are in the image of $\Gamma_{g,(k), 1}$ for some large $g$. Now choose an appropriate diffeomorphism of the surface $S_{2 g, k+1}$ which moves the odd genus over the even genus to the last $g$ genus. Let $c \in \Gamma_{\infty,(k)}$ be its homotopy class. By Equation (2.3), $c$ and $d$ are in the maximal perfect subgroups of $\Gamma_{\infty,(k)}$ and $\Gamma_{\infty}$ respectively. On the other hands, the map $l$ mentioned above is a splitting homomorphism, and clearly the homotopy fiber of the map $l^{+}: B \Gamma_{\infty,(k)}^{+} \rightarrow B \Gamma_{\infty}^{+}$is $B \Sigma_{k}^{+}$. Hence, by Corollary 2.10, we have

$$
\left(B \Gamma_{\infty,(k)}\right)^{+} \simeq B \Gamma_{\infty}^{+} \times B \Sigma_{k}^{+} .
$$

REMARK 2.12. Bödigheimer [2] proved the following general case:

$$
H_{*}\left(\Gamma_{g,(n), m}^{(k)} ; \mathbb{F}\right) \simeq H_{*}\left(\Gamma_{g, 1} ; \mathbb{F}\right) \otimes H_{*}\left(\Sigma_{n} ; \mathbb{F}\right) \otimes H_{*}\left(\Sigma_{k} ; \mathbb{F}\left[x_{1}, \ldots, x_{k}\right]\right),
$$

where $* \leq \frac{g}{2}, k+n+m \geq 1, \mathbb{F}$ is any field.

\section{A Harer map and its homology triviality}

We discuss a map from the braid group to the mapping class group which is defined geometrically, i.e., by identifying the braid group as a subgroup of the mapping class group of a surface. 
The basic idea is to identify the braid group as a subgroup of the mapping class group of a genus zero surface with boundary components as follows. Let $S_{0, k+1}$ be a sphere with $k+1$ disks removed and parametrized boundary circles $\partial_{0}, \partial_{1}, \ldots, \partial_{k}$. Consider the orientation preserving diffeomorphisms that fix the first boundary component $\partial_{0}$ pointwise but may permute the other $k$ boundary components as long as they preserve the parametrization of each. The associated mapping class group $\Gamma_{0,(k), 1}$ is the ribbon braid group $R B_{k}$ on $k$ ribbons. $R B_{k}$ is the wreath product $B_{k} \imath \mathbb{Z}$, and $B_{k}$ can naturally be identified as a subgroup.

$$
\Gamma_{0,1}^{k} \simeq B_{k} \subset B_{k} \prec \mathbb{Z}=R B_{k} \simeq \Gamma_{0,(k), 1} .
$$

Thus this identification leads to homomorphisms of the braid group into the mapping class group. We define a Harer map as follows:

Consider two copies of the surface $S_{0, k+1}$ glued along their boundary components $\partial_{1}, \ldots, \partial_{k}$ to form a surface $S_{k-1,2}$ (See Figure 4 ).

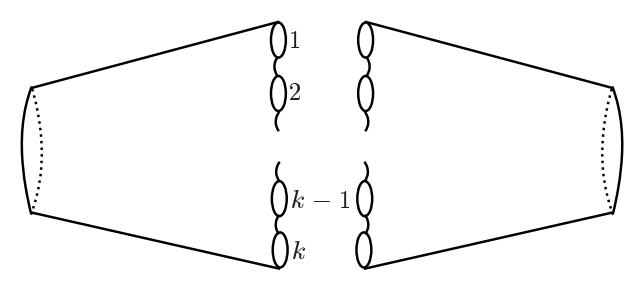

FiguRE $4 . S_{0, k+1} \longrightarrow S_{k-1,2}$

Any diffeomorphism of $S_{0, k+1}$ as described above can be extended to $S_{k-1,2}$ by "mirroring" the action on the second copy of $S_{0, k+1}$ and can then be extended to $S_{g+k, 2}$ by the identity diffeomorphism (See Figure $5)$.

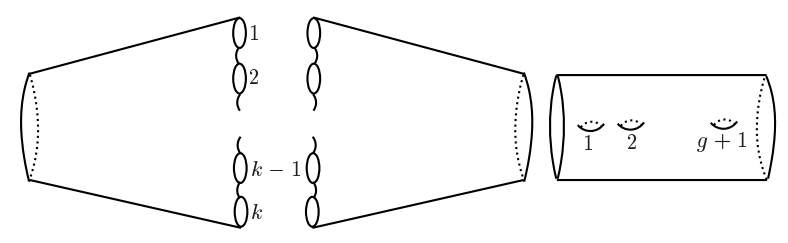

FIGURE 5. $S_{0, k+1} \longrightarrow S_{g+k, 2}$ 
This gives rise to a Harer map:

$$
\text { Harer : } B_{k} \stackrel{m}{\longrightarrow} B_{k} \times_{\Sigma_{k}} B_{k} \stackrel{\alpha}{\longrightarrow} \Gamma_{g+k, 2},
$$

where $m$ is mirroring, $\alpha$ is induced by gluing $S_{g+1,2}$ on the right boundary component of $S_{k-1,2}$, and the group in the middle is defined as the pullback in the following diagram:

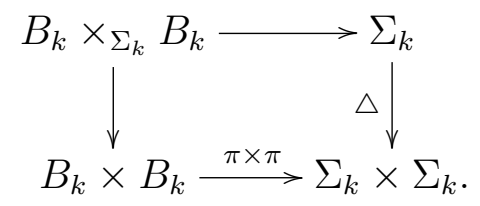

Here, $\triangle$ denotes the diagonal map, and $\pi: B_{k} \rightarrow \Sigma_{k}$ is the canonical surjection.

We will consider a homomorphism from the braid group to the mapping class group, which factors through the symmetric group. In the sequel, we will call this map as a Harer map.

We now prove the following theorem:

Main Theorem. The image of $\operatorname{Harer}_{*}: H_{*}\left(B_{k} ; \mathbb{F}\right) \rightarrow H_{*}\left(\Gamma_{g+k, 2} ; \mathbb{F}\right)$ is zero for $0<*<\frac{g+k}{2}$ and $\mathbb{F}=\mathbb{Q}, \mathbb{Z}_{p}(p \neq 2$ is prime). Hence the map Harer $_{*}: H_{*}\left(B_{\infty} ; \mathbb{F}\right) \rightarrow H_{*}\left(\Gamma_{\infty, 1} ; \mathbb{F}\right)$ is zero.

Proof. Consider the following commutative diagram of group homomorphisms

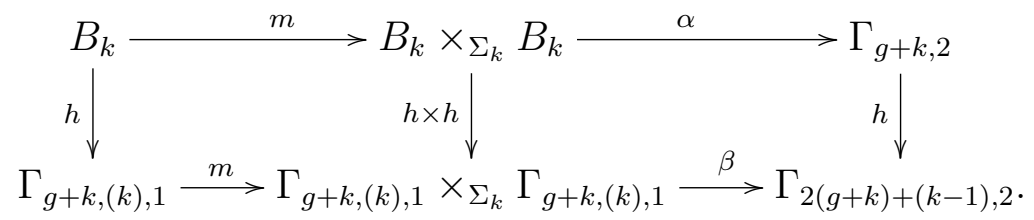

Here, $\Gamma_{g+k,(k), 1}$ denotes the mapping class group of the surface $S_{g+k, k+1}$, where $k$ of the boundary components may be permuted as long as the parametrization of each component is preserved while one of the boundary components is fixed pointwise; the group in the middle on the bottom is defined as a pull-back as above. The left vertical map $s$ is induced by gluing one of the boundary components of $S_{g+k, 2}$ along $\partial_{0}$ to a copy of $S_{0, k+1}$. The right vertical map $h$ is induced by gluing one of the boundary components of $S_{g+2 k-1,2}$ along $\partial_{0}$ to a copy of $S_{g+k, 2}$. The left horizontal maps $m$ are defined by "mirroring", while the bottom right horizontal map $\beta$ is defined by identifying the two copies of the boundary components $\partial_{1}, \ldots, \partial_{k}$. 
Consider the following commutative diagram:

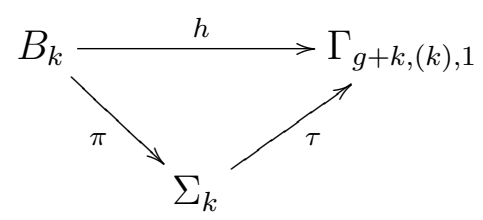

Here, $\tau$ denotes the action on the $k$ boundary components of $S_{g+k,(k), 1}$. Thus, we have the following commutative diagram:

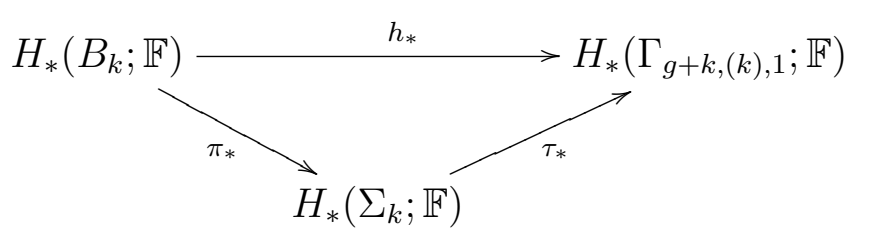

By Theorem 2.11 and (2.1), we have

$$
H_{*}\left(\Gamma_{g+k,(k), 1} ; \mathbb{F}\right) \simeq H_{*}\left(\Gamma_{g+k, 1} ; \mathbb{F}\right) \otimes H_{*}\left(\Sigma_{k} ; \mathbb{F}\right)
$$

for $* \leq \frac{g+k-1}{2}, \mathbb{F}$ is any field.

So, the map $h: B_{k} \rightarrow \Gamma_{g+k,(k), 1}$ factors in homology in degrees $*<\frac{g+k}{2}$ through $\Sigma_{k}$. Hence, the map Harer $: H_{*}\left(B_{\infty} ; \mathbb{F}\right) \rightarrow H_{*}\left(\Gamma_{\infty, 1} ; \mathbb{F}\right)^{2}$ is determined by the following commutative diagram:

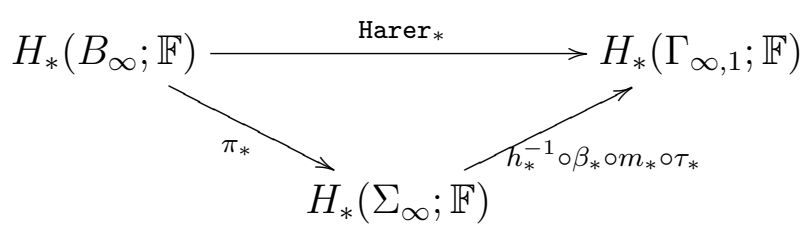

Now, we consider the following two cases.

Case $1(\mathbb{F}=\mathbb{Q})$ : By Corollary $2.4, H_{*}\left(\Sigma_{n} ; \mathbb{Q}\right)=0$; In fact, by Universal Coefficients Theorem for Homology, we also have the same result:

$$
\begin{aligned}
H_{1}\left(\Sigma_{\infty} ; \mathbb{Q}\right) & \simeq\left(H_{1}\left(\Sigma_{\infty} ; \mathbb{Z}\right) \otimes \mathbb{Q}\right) \oplus \operatorname{Tor}\left(H_{0}\left(\Sigma_{\infty} ; \mathbb{Z}\right), \mathbb{Q}\right) \\
& \simeq H_{1}\left(\Sigma_{\infty} ; \mathbb{Z}\right) \otimes \mathbb{Q} \\
& \simeq \mathbb{Z}_{2} \otimes \mathbb{Q} \\
& \simeq 0
\end{aligned}
$$

Thus the image of Harer $: H_{*}\left(B_{k} ; \mathbb{Q}\right) \rightarrow H_{*}\left(\Gamma_{g+k, 2} ; \mathbb{Q}\right)$ is zero for $0<$ $*<\frac{g+k}{2}$. 
Case $2\left(\mathbb{F}=\mathbb{Z}_{p}, p \neq 2\right.$ is prime): By Universal Coefficients Theorem for Homology, we get the following:

$$
\begin{aligned}
H_{1}\left(\Sigma_{\infty} ; \mathbb{Z}_{p}\right) & \simeq\left(H_{1}\left(\Sigma_{\infty} ; \mathbb{Z}\right) \otimes \mathbb{Z}_{p}\right) \oplus \operatorname{Tor}\left(H_{0}\left(\Sigma_{\infty} ; \mathbb{Z}\right), \mathbb{Z}_{p}\right) \\
& \simeq H_{1}\left(\Sigma_{\infty} ; \mathbb{Z}\right) \otimes \mathbb{Z}_{p} \\
& \simeq \mathbb{Z}_{2} \otimes \mathbb{Z}_{p} \\
& \simeq \begin{cases}0 & \text { for } p \neq 2 \\
\mathbb{Z}_{2} & \text { for } p=2 .\end{cases}
\end{aligned}
$$

It is also well known $([3,11])$ that the surjection $B_{n} \rightarrow \Sigma_{n}$ induces on group completions up to homotopy the inclusion map $\Omega^{2} S^{2} \rightarrow \Omega^{\infty} S^{\infty}$.

Recall that F. Cohen in [3] describes the homology of the braid group with $\mathbb{Z}_{p}$ coefficients for every prime $p$ in terms of a one-dimensional generator $x_{1} \in H_{1}\left(B_{\infty} ; \mathbb{Z}_{p}\right)$ and powers of homology operations (DyerLashof homology operations) applied to $x_{1}$. On the other hand,

$$
H_{1}\left(\text { Aut }_{\infty} ; \mathbb{Z}\right)=H_{1}\left(\Sigma_{\infty} ; \mathbb{Z}\right)=\mathbb{Z}_{2},
$$

and hence it follows that $\pi_{*}$ is zero in all positive dimensions for all odd $p$.

The right vertical map $h_{*}$ is a homology isomorphism in theses degrees by the stability theorem of Harer (Theorem 2.1). Thus by Cases 1 and 2, the image of $\operatorname{Harer}_{*}: H_{*}\left(B_{k} ; \mathbb{F}\right) \rightarrow H_{*}\left(\Gamma_{g+k, 2} ; \mathbb{F}\right)$ is zero for $0<*<\frac{g+k}{2}$, $\mathbb{F}=\mathbb{Q}, \mathbb{Z}_{p}$

REMARK 3.1. Since $H_{*}\left(\Sigma_{\infty} ; \mathbb{Z}\right) \simeq \mathbb{Z}_{2}$, the image of $\pi: B_{k} \rightarrow \Sigma_{k}$ in homology contains only 2 -torsion for $0<*<\frac{g+k}{2}$. Hence we would also expect that the image of $\operatorname{Harer}_{*}: H_{*}\left(B_{k} ; \mathbb{Z}\right) \rightarrow H_{*}\left(\Gamma_{g+k, 2} ; \mathbb{Z}\right)$ contains at most 2 -torsion for $0<*<\frac{g+k}{2}$.

\section{References}

[1] E. Artin, Theorie der Zöpfe, Abh. Math. Sem. Univ. Hamburg 4 (1926), 47-72.

[2] C.-F. Bödigheimer and U. Tillmann, Stripping and splitting decorated mapping class groups, Cohomological methods in homotopy theory (Bellaterra, 1998), 47-57, Progr. Math., 196, Birkhauser, Basel, 2001.

[3] F. R. Cohen, T. J. Lada, and J. P. May, The homology of iterated loop space, Lecture Notes in Mathematics 533, Springer-Verlag, 1976.

[4] S. Galatius, Stable homology of automorphism groups of free groups, Ann. of Math. (2) 173 (2) (2011), 705-768.

[5] J. Harer, Stability of the homology of the mapping class groups of orientable surfaces, Ann. of Math. (2) 121 (2) (1985), 215-249. 
[6] A. Hatcher and K. Vogtmann, Cerf theory for graphs, J. London Math. Soc. (2) 58 (3) (1998), 633-655.

[7] _ Homology stability for outer automorphism groups of free groups, Algebr. Geom. Topol. 4 (2004), 1253-1272.

[8] N. V. Ivanov, Stabilization of the homology of Teichmüller modular groups, Algebra i Analiz 1 (3) (1989), 110-126; Leningrad Math. J. 1 (3) (1990), 675-691.

[9] M. Nakaoka, Decomposition theorem for homology groups of symmetric groups, Ann. of Math. (2) 71 (1960), 16-42.

[10] J. Powell, Two theorems on the mapping class group of a surface, Proc. Amer. Math. Soc. 68 (3) (1978), 347-350.

[11] G. Segal, Configuration spaces and iterated loop spaces, Invent. Math. 21 (1973), 213-221.

[12] U. Tillmann, Artin's map in stable homology, Bull. Lond. Math. Soc. 39 (6) (2007), 989-992.

[13] J. B. Wagoner, Delooping classifying spaces in algebraic K-theory, Topology 11 (1972), 349-370.

Departments of Mathematics

Inha University

Incheon 402-751, Korea

E-mail: clique5132@hanmail.net

Departments of Mathematics

Inha University

Incheon 402-751, Korea

E-mail: yjsong@inha.ac.kr 